\title{
Probing many-body states of ultracold atoms via noise correlations
}

\section{Citation}

Altman, Ehud, Eugene Demler, and Mikhail D. Lukin. 2004. “Probing Many-Body States of Ultracold Atoms via Noise Correlations." Physical Review A 70 (1) (July 6). doi:10.1103/ physreva.70.013603.

\section{Published Version}

doi:10.1103/physreva. 70.013603

\section{Permanent link}

http://nrs.harvard.edu/urn-3:HUL.InstRepos:27945233

\section{Terms of Use}

This article was downloaded from Harvard University's DASH repository, and is made available under the terms and conditions applicable to Other Posted Material, as set forth at http:// nrs.harvard.edu/urn-3:HUL.InstRepos:dash.current.terms-of-use\#LAA

\section{Share Your Story}

The Harvard community has made this article openly available.

Please share how this access benefits you. Submit a story.

Accessibility 


\title{
Probing many-body states of ultracold atoms via noise correlations
}

\author{
Ehud Altman, Eugene Demler, and Mikhail D. Lukin \\ Physics Department, Harvard University, Cambridge, Massachusetts 02138, USA
}

(Received 10 June 2003; published 6 July 2004)

\begin{abstract}
We propose to utilize density-density correlations in the image of an expanding gas cloud to probe complex many-body states of trapped ultracold atoms. In particular, we show how this technique can be used to detect superfluidity of fermionic gases and to study spin correlations of multicomponent atoms in optical lattices. The feasibility of the method is investigated by analysis of the relevant signal to noise ratio including experimental imperfections.
\end{abstract}

DOI: $10.1103 /$ PhysRevA.70.013603

PACS number(s): 03.75.Ss, 03.75.Mn, 42.50.Lc

Much of the excitement in the field of Bose-Einstein condensation (BEC) is due to the clear demonstration it provides of the wave character of matter. The condensed state of bosons involves macroscopic occupation of a delocalized single particle state. Consequently, it is characterized by sharp density peaks in the freely expanding gas cloud after it is released from the trap [1]. Patterns that appear when two or more superfluid clouds interfere [2], are a direct probe of the single particle coherence, amplified by macroscopic occupation.

Recent experiments open intriguing directions for studying many-body phenomena beyond single particle coherence. For example, observation of the superfluid to Mott-insulator transition [3], as well as experiments involving ultracold fermions near a Feshbach resonance [4], address strongly correlated states of matter. The most intriguing aspect of such systems is the existence of nontrivial correlations and complex order that defy a description in terms of (single particle) matter waves. Accordingly, they cannot be characterized simply by the density profile of an expanding cloud. For example, the localized atoms in Mott states of the optical lattice display a vanishing interference pattern [3], which can hardly reveal detailed properties of the quantum state. Likewise, superfluidity of paired fermions is not evident as a coherence peak in the density profile [5], and detecting the order parameter presents a considerable challenge. Observation of some theoretically proposed "exotic" many-atom states $[6,8,9]$ may prove even more elusive.

In this letter, we show that the quantum nature of certain strongly correlated states can be revealed by spatial noise correlations in the image of the expanding gas. This is similar in spirit to measurements of nonclassical correlations of light in optical systems [11] and temporal current noise in mesoscopic conductors [10]. In analogy to quantum optics, this technique allows us to study matter waves that lack single particle coherence. Specifically we show: (i) Fermionic atoms released from the trap would display a clear signature of superfluidty in their density correlations. Furthermore, detailed properties of the fermionic superfluidity can be studied, such as pairing symmetry, and BCS to BEC crossover [12]; (ii) Atoms released from a Mott-insulating state of the optical lattice display sharp (Bragg) peaks in the density-density correlation function as a consequence of quantum statistics; (iii) These peaks can be used to probe spin correlations of multicomponent bosons on optical lat- tices. In particular, spin ordered Mott states proposed for two-component bosons [7,8] can be detected. Finally, we verify the experimental feasibility of the proposed measurements.

Before proceeding, we note that earlier proposals to detect fermionic superfluidity relied on dynamical response of the cloud [13], inelastic scattering of light to induce and measure excitations $[14,16]$, or to microscopically image the pair wave function in the trap [15]. In contrast, the present technique provides a direct probe of the pair coherence as well as information on the pairing symmetry. We point out a recent experiment that analyzed real-space density correlations in interfering copies of a condensate, split by a Bragg pulse [17].

We proceed by formulating a detection scheme for atoms released from a single macroscopic trap. Suppose, for simplicity, that the system is initially in some pure state $|\Phi\rangle$. In a typical experimental setup, the trapping potential is turned off suddenly, and the atoms evolve independently under the influence of the free propagator $U_{0}(t)$. This is valid provided that the free-atom collision cross section is not too large. Such conditions can be achieved for example by switching the magnetic field to values far from the Feshbach resonance when turning off the trap [4].

In such time of flight experiments, the column integrated density of the expanding cloud is measured by light absorption imaging [18]. The images are commonly analyzed by comparing to theoretical predictions for the density expectation value, given by

$$
\left\langle\hat{n}_{\alpha}(\mathbf{r})\right\rangle_{t}=\left\langle\Phi\left|U_{0}^{\dagger}(t) \psi_{\alpha}^{\dagger}(\mathbf{r}) \psi_{\alpha}(\mathbf{r}) U_{0}(t)\right| \Phi\right\rangle,
$$

where $\psi_{\alpha}$ is the field operator for bosons or fermions and $\alpha$ denotes an internal atomic quantum number (spin). At finite temperature, the expectation value in (1) is replaced by a thermal average. After a long time of flight, the density distribution becomes proportional to the momentum distribution in the initial trapped state $\langle n(\mathbf{r})\rangle_{t} \approx(m / h t)\left\langle\hat{n}_{\mathbf{Q}(\mathbf{r})}\right\rangle$. The wave vector $\mathbf{Q}(\mathbf{r})=m \mathbf{r} /(\hbar t)$ defines a correspondence between position in the cloud and momentum in the trap.

It is important to realize that in each experimental image, a single realization of the density is observed, not the expectation value. Equation (1) is still meaningful, because the density is a self-averaging quantity. Each bin $\sigma$ in the image 
represents a substantial number of atoms $N_{\sigma}$, while the atomic noise scales as $O\left(\sqrt{N_{\sigma}}\right)$. However, since $N_{\sigma}$ is not macroscopic, the density fluctuations are visible. They are characterized by the correlation function

$$
\mathcal{G}_{\alpha \beta}\left(\mathbf{r}, \mathbf{r}^{\prime}\right)=\left\langle\hat{n}_{\alpha}(\mathbf{r}) \hat{n}_{\beta}\left(\mathbf{r}^{\prime}\right)\right\rangle_{t}-\left\langle\hat{n}_{\alpha}(\mathbf{r})\right\rangle_{t}\left\langle\hat{n}_{\beta}\left(\mathbf{r}^{\prime}\right)\right\rangle_{t} .
$$

In analogy with Eq. (1) this can be related to ground state momentum correlations

$$
\mathcal{G}_{\alpha \beta}\left(\mathbf{r}, \mathbf{r}^{\prime}\right) \propto\left\langle\hat{n}_{\mathbf{Q}(\mathbf{r}) \alpha} \hat{n}_{\mathbf{Q}\left(\mathbf{r}^{\prime}\right) \beta}\right\rangle-\left\langle\hat{n}_{\mathbf{Q}(\mathbf{r}) \alpha}\right\rangle\left\langle\hat{n}_{\mathbf{Q}\left(\mathbf{r}^{\prime}\right) \beta}\right\rangle .
$$

The proportionality constant is $(m / \hbar t)^{2 d}$, where $d$ is the dimensionality. We shall be concerned primarily with pure density-density correlations $\mathcal{G}\left(\mathbf{r}, \mathbf{r}^{\prime}\right)=\Sigma_{\alpha \beta} \mathcal{G}_{\alpha \beta}\left(\mathbf{r}, \mathbf{r}^{\prime}\right)$, which do not require state-selective measurement. In practice, it may be more convenient to consider the quantity $\Delta n\left(\mathbf{r}, \mathbf{r}^{\prime}\right)$ $\equiv n(\mathbf{r})-n\left(\mathbf{r}^{\prime}\right)$, whose fluctuations are closely related to $\mathcal{G}\left(\mathbf{r}, \mathbf{r}^{\prime}\right)$. If $\langle n(\mathbf{r})\rangle_{t}=\left\langle n\left(\mathbf{r}^{\prime}\right)\right\rangle_{t}$, then

$$
\left\langle\Delta n\left(\mathbf{r}, \mathbf{r}^{\prime}\right)^{2}\right\rangle_{t}=\mathcal{G}(\mathbf{r}, \mathbf{r})+\mathcal{G}\left(\mathbf{r}^{\prime}, \mathbf{r}^{\prime}\right)-2 \mathcal{G}\left(\mathbf{r}, \mathbf{r}^{\prime}\right) .
$$

Fermionic superfluids. As a specific example, we now consider superfluid states of fermionic atoms. Such superfluids sustain macroscopic coherence and their transport properties are similar to their bosonic counterparts. However, the average density profile of the expanding cloud would not reveal their superfluid nature. The second-order coherence (3), on the other hand, is a direct probe of pair correlations in momentum space. It is therefore particularly adept to detect the condensed state of fermions, which is defined by a macroscopic occupation of zero-momentum pairs. This would be seen as pronounced correlations of density fluctuations between diametrically opposite sides of the cloud.

As an illustration consider first a system at zero temperature described by a BCS-like ground state

$$
\left|\Phi_{B C S}\right\rangle=\prod_{k}\left(u_{\mathbf{k}}+v_{\mathbf{k}} a_{\mathbf{k} \uparrow}^{\dagger} a_{-\mathbf{k} \downarrow}^{\dagger}\right)|0\rangle .
$$

Note that this wave function can describe weak, as well as tightly bound pairs for which $u_{\mathbf{k}}$ and $v_{\mathbf{k}}$ have a wide momentum distribution [12]. The average density profile of the expanding cloud (1) is proportional to the BCS momentum distribution function $\langle\hat{n}(\mathbf{r})\rangle_{t}=2\left|v_{\mathbf{Q}(\mathbf{r})}\right|^{2}$, which is qualitatively indistinguishable from a Fermi distribution at $T=T_{C}$ [4].

The essential difference between these states lies in the two-particle correlations. For every atom with momentum k in the BCS state, there is another one at exactly -k. Indeed, a straightforward application of (3) for the BCS state gives

$$
\mathcal{G}\left(\mathbf{r}, \mathbf{r}^{\prime}\right)=n_{s}(\mathbf{r}) \tilde{\delta}\left(\mathbf{r}+\mathbf{r}^{\prime}\right)
$$

where $n_{s}(\mathbf{r})=2\left|u_{\mathbf{Q}(\mathbf{r})}\right|^{2}\left|v_{\mathbf{Q}\left(\mathbf{r}^{\prime}\right)}\right|^{2} . \tilde{\delta}\left(\mathbf{r}+\mathbf{r}^{\prime}\right)$ is a sharply peaked function of $\mathbf{r}+\mathbf{r}^{\prime}$, a direct analogue of the first-order coherence peak signaling BEC. In a finite system, the peak width $\sim \hbar t / m L$ is set by the spatial extent of the system, while its precise form depends on the details of the superfluid wave function in the trap. However, $n_{s}(\mathbf{r})$ the weight of the peak at $\mathbf{r}+\mathbf{r}^{\prime}=0$, is of completely general applicability. It simply counts the number of zero momentum pairs with specified individual momenta. The angular average of $n_{s}(\mathbf{r})$ gives the
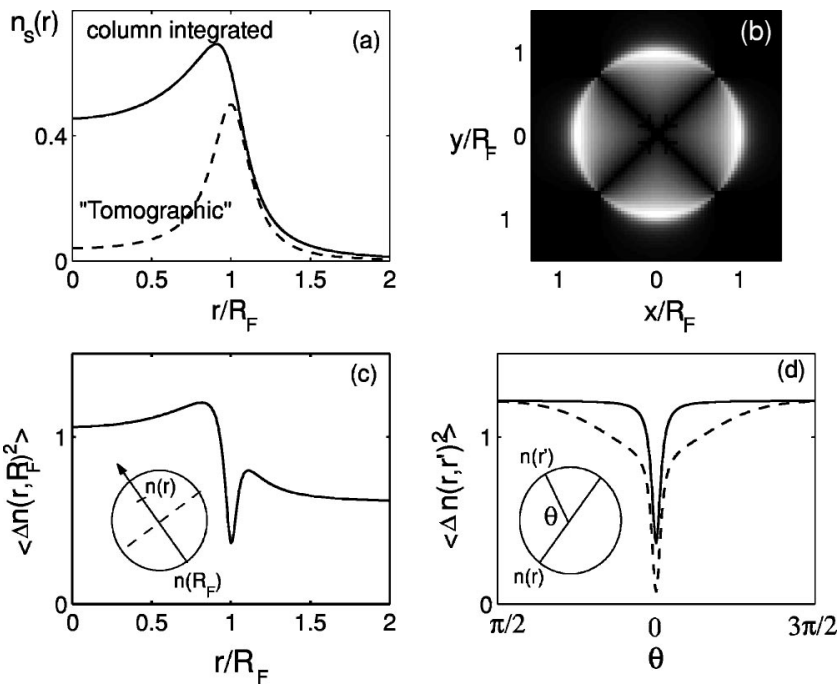

FIG. 1. Fermionic superfluid. (a) Angle integrated weight of the correlation peak at diametrically opposite points. Solid line is the column integrated result relevant to experiment. (b) Weight of the correlation peak for $d_{x^{2}-y^{2}}$ pairing, which may be expected in a realization of the Hubbard model on an optical lattice. (c) and (d) depict $\left\langle\Delta n\left(\mathbf{r}, \mathbf{r}^{\prime}\right)^{2}\right\rangle$ at $T>0$ (superfluid fraction 0.7 ). $\mathbf{r}$ is fixed on the Fermi surface. In (c) $\mathbf{r}^{\prime}$ is varied along the same diameter while in (d) $\mathbf{r}^{\prime}$ it is varied around the Fermi surface so that the relative angle between $\mathbf{r}$ and $\mathbf{r}^{\prime}$ is $\theta$. Dashed line corresponds to BEC of tightly bound pairs, whereas the solid line is the BCS limit at the same temperature. The width of the narrow dip $\sim \hbar t /(m L)$ is limited by the system's size.

radial part of the pairing wave function. This is plotted for the BCS state in Fig. 1(a) (dashed). The solid line as well as other plots in Fig. 1 depict the experimentally observable column integrated functions.

Pairing symmetries other than $s$-wave, would be detected by higher angular harmonics in the pair distribution $n_{s}(\mathbf{r})$. The ability to detect $d_{x^{2}-y^{2}}$ pairing [Fig. 1(b)] is of particular interest in light of recent proposals to realize a Hubbard model of fermions on an optical lattice [14]. Such experiments may resolve a key issue in the understanding of high $T_{C}$ superconductivity in cuprates.

In practice, it may be more convenient to measure the fluctuations of $\Delta n\left(\mathbf{r}, \mathbf{r}^{\prime}\right)$ which does not require exact knowledge of the average. Noting that the state (5) is an eigenstate of $\Delta n(\mathbf{r},-\mathbf{r})$ with eigenvalue zero, we have $\left\langle\Delta n(\mathbf{r},-\mathbf{r})^{2}\right\rangle$ $\equiv 0$ at $T=0$. Well away from $\mathbf{r}+\mathbf{r}^{\prime}=0\left\langle\Delta n\left(\mathbf{r}, \mathbf{r}^{\prime}\right)^{2}\right\rangle \sim n_{\mathbf{Q}(\mathbf{r})}(1$ $\left.-n_{\mathbf{Q}(\mathbf{r})}\right)+n_{\mathbf{Q}\left(\mathbf{r}^{\prime}\right)}\left(1-n_{\mathbf{Q}\left(\mathbf{r}^{\prime}\right)}\right)$, which is nonzero on the smeared Fermi surface of a Fermi liquid (at $T>0$ ) and for a BCS state. A sharp dip, whose width $\hbar t /(m L)$ is limited by the system size, appears around $\mathbf{r}^{\prime}=\mathbf{r}$ in the superfluid state [Figs. 1(c) and 1(d)].

How the correlation dip changes when the temperature is raised, depends on the type of low energy excitations available to the system. In a weakly coupled BCS superfluid ( $\Delta$ $\left.\ll \epsilon_{F}\right)$, quasiparticle excitations are dominant at low temperatures. Since these are pair breaking excitations, the pairing dip will gradually diminish with temperature and finally vanish at $T=T_{c}$. At strong coupling on the other hand, the domi- 
nant low energy excitations are collective BogoliubovAnderson modes. These describe bound pairs, excited to nonzero center of mass momentum. In direct analogy with BEC, we expect a wide background dip associated with pairing correlations at nonzero center of mass momentum. Thus, the behavior of the correlation dip at $T>0$, can be used as a probe of the pairing regime. Here we assume that even at strong pairing, close to a Feshbach resonance, the atoms expand without interactions once the trap is shut off. One can avoid collisions in the expansion by tuning the magnetic field away from resonance, or by transferring the atoms into another pair of hyperfine sublevels upon release. The sudden approximation, implicit in formulas (3) and (4), holds provided the change is applied over a sufficiently short time scale $\tau \ll \hbar / \Delta$, where $\Delta$ is the pairing gap.

Optical lattice. We now consider atoms initially confined to an optical lattice. Calculation of $\langle n(\mathbf{r})\rangle$ and $\left\langle n(\mathbf{r}) n\left(\mathbf{r}^{\prime}\right)\right\rangle$ involves operating on the lattice state $|\Phi\rangle$ with $U(t)^{\dagger} \psi(\mathbf{r}) U(t)$. In normal ordered expectation values, this operator can be safely replaced by its projection into the lowest Bloch band, $A(\mathbf{r}, t) \equiv \sum_{i} w_{i}(\mathbf{r}, t) a_{i \alpha}[19]$. Here, $w_{i}(\mathbf{r}, t)$ is the free evolution of a Wannier wave function, initially centered around the lattice site at $R_{i}$ and $a_{i \alpha}=\int d \mathbf{r} w_{i}(\mathbf{r}, 0) \psi_{\alpha}(\mathbf{r})$ is a particle annihilation operator at this site. At long times $w_{i}(\mathbf{r}, t) \propto e^{-i \mathbf{Q}(\mathbf{r}) \cdot \mathbf{R}_{i}}$ times a Gaussian envelope, which we shall approximate by a square of the same width $W=\hbar t /\left(a_{0} m\right) . a_{0}$ is the width of the Wannier state on the lattice. Now, $\left\langle\hat{n_{\alpha}}(\mathbf{r})\right\rangle_{t}$ can be related to the off-diagonal correlation function in the ground state [19]

$$
\left\langle\hat{n}_{\alpha}(\mathbf{r})\right\rangle_{t} \approx\left\langle\hat{n}_{\mathbf{Q}(\mathbf{r})}\right\rangle \equiv \frac{1}{W^{d}} \sum_{i, j} e^{i\left(\mathbf{R}_{i}-\mathbf{R}_{j}\right) \cdot \mathbf{Q}(\mathbf{r})}\left\langle a_{i \alpha}^{\dagger} a_{j \alpha}\right\rangle .
$$

The wave vector $\mathbf{Q}(\mathbf{r})$ now defines a correspondence between position in the cloud and the quasimomentum on the lattice. $\mathcal{G}$ can also be written in terms of correlations in the lattice state:

$$
\begin{aligned}
\mathcal{G}_{\alpha, \beta}\left(\mathbf{r}, \mathbf{r}^{\prime}\right) \sim & \frac{1}{W^{2 d}} \sum_{i i^{\prime} j j^{\prime}} e^{i \mathbf{R}_{i i^{\prime}} \cdot \mathbf{Q}(\mathbf{r})+i \mathbf{R}_{j j^{\prime}} \cdot \mathbf{Q}\left(\mathbf{r}^{\prime}\right)}\left\langle a_{i \alpha}^{\dagger} a_{j \beta}^{\dagger} a_{j^{\prime} \beta} a_{i^{\prime} \alpha}\right\rangle \\
& +\delta_{\alpha \beta} \delta\left(\mathbf{r}-\mathbf{r}^{\prime}\right)\left\langle n_{\alpha}(\mathbf{r})\right\rangle_{t}-\left\langle n_{\alpha}(\mathbf{r})\right\rangle\left\langle n_{\beta}\left(\mathbf{r}^{\prime}\right)\right\rangle .
\end{aligned}
$$

Here, $\delta\left(\mathbf{r}-\mathbf{r}^{\prime}\right)$ is a true $\delta$-function originating from normal ordering in the continuum. Note that formulas (7) and (8) hold for bosons and fermions.

In the superfluid state of bosons, where $\left\langle a_{i}^{\dagger} a_{j}\right\rangle=|\Psi|^{2}$, $n(\mathbf{r}, t)$ exhibits Bragg peaks at $\mathbf{Q}(\mathbf{r})$ corresponding to reciprocal lattice vectors $\mathbf{G}$. In the Mott state, on the other hand, $\left\langle a_{i}^{\dagger} a_{j}\right\rangle \approx \delta_{i j}$, and there is no interference pattern in $n(\mathbf{r}, t)$.

The Mott state, however, displays nontrivial correlations in the second-order correlation function (8) associated with atom number fluctuations. To illustrate their origin, and to make contact with the Hanbury-Brown-Twiss effect in quantum optics, we first apply (7) and (8) to the "toy" model of two atoms localized in two wells. For an initial Fock state $|\Phi\rangle=a_{1}^{\dagger} a_{2}^{\dagger}|0\rangle$ it gives:
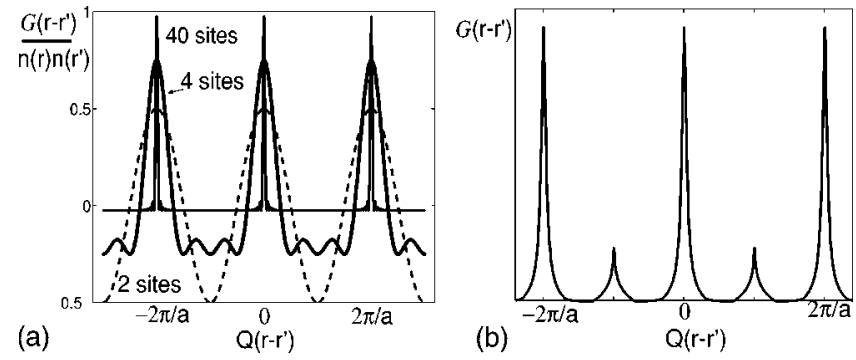

FIG. 2. (a) Normalized density correlations, in the Mott state for a chain of 2, 4, and 40 sites. (b) Density correlations for an antiferromagnetic Mott chain of two-component bosons. The weak singularities (broad peaks) reflect power-law correlations in the onedimensional system.

$$
\mathcal{G}\left(\mathbf{r}, \mathbf{r}^{\prime}\right)=\frac{2}{W^{2 d}} \eta \cos \left[\frac{m}{\hbar t}\left(\mathbf{r}-\mathbf{r}^{\prime}\right)\right],
$$

where $\eta=1(-1)$ for bosons (fermions). The oscillations reflect a two-body interference effect that amounts to bunching in the case of bosons and antibunching for fermions, as in the textbook example $[11,20]$. Figure 2 shows how these oscillations develop into coherent Bragg peaks with increasing lattice size. A fermionic insulating state will display similar evolution with peaks replaced by dips.

Note that the Bragg peaks in the second-order coherence do not reflect reduced number fluctuations in the Mott state, but rather the permutation symmetry of the wave function. In particular, a thermal Bose gas on a lattice would exhibit the same coherent peaks. However, in a Mott state with nontrivial internal structure, the noise correlations can reveal complex order, as we now illustrate.

Let us consider for example Mott states of twocomponent bosons (or fermions) with exactly one atom per site. Since the motional degrees of freedom are frozen, (8) can be written in terms of a pseudospin correlation function. In particular, the pure density-density correlations in the cloud are given by

$$
\begin{aligned}
\mathcal{G}\left(\mathbf{r}, \mathbf{r}^{\prime}\right)= & \frac{\eta}{2} \frac{N}{W^{d}}\left(\frac{2 \pi a_{0}}{l}\right)^{d} \sum_{\mathbf{G}} \widetilde{\delta}^{d}\left(\mathbf{r}-\mathbf{r}^{\prime}+\frac{\hbar t}{m} \mathbf{G}\right) \\
& +2 \eta \sum_{i j} e^{i\left[\mathbf{Q}(\mathbf{r})-\mathbf{Q}\left(\mathbf{r}^{\prime}\right)\right] \cdot \mathbf{R}_{i j}\left\langle\mathbf{S}_{i} \cdot \mathbf{S}_{j}\right\rangle,}
\end{aligned}
$$

where $\mathbf{S}_{i}=\frac{1}{2} a_{\alpha i}^{\dagger} \vec{\sigma}_{\alpha \beta} a_{\beta i}$ are the pseudospin- $\frac{1}{2}$ operators, $\eta=$ $+(-) 1$ for bosons (fermions), $l$ is the lattice spacing and $N$ the number of sites. This expression constitutes one of our main results. While the first term in (10) is just a remaining signature of quantum statistics, the second corresponds to the static spin structure factor. Thus observation of density correlations in the expanding cloud provides experimental access to the spin correlation function in the trap. Indeed, various possible spin orders have been predicted for twocomponent systems $[7,8]$ that may be probed using our method. Figure 2 illustrates this for the case of a onedimensional antiferromagnet, characterized by weak singularities [logarithmic in case of $S U(2)$ symmetry] in the density correlations at half the reciprocal lattice vectors. 
Antiferromagnetism in higher dimensions would be seen as sharp Bragg peaks at these points. A counterflow superfluid $[7,8]$, characterized by $x-y$ ferromagnetic order, can be detected by applying a magnetic field gradient shifting the correlations to a finite wave vector.

Experimental issues. To observe the proposed effects, two criteria must be met: (i) the atomic noise should be observable in an experiment and (ii) correlated fluctuations should rise, in a statistical sense, above the uncorrelated noise.

In a typical experiment, the cloud is integrated over narrow cylinders whose bases $\sigma$ correspond to the spatial resolution. The noise of detected probe-photons in a bin of area $\sigma$ is a sum of contributions from atomic and laser light fluctuations. The latter is fundamentally limited by photon shot noise. The atomic noise exceeds the photon shot noise, provided the number of photons per bin in the incoming pulse, $p_{\sigma}>\exp (2 \kappa)\left\langle N_{\sigma}\right\rangle /\left(\eta \kappa^{2}\right)$, where $\kappa$ is the optical depth of the cloud, which should be chosen close to unity, and $\eta$ is the photon detection efficiency. The practical limitation on the number of photons is associated with photon recoil, which results in image blurring. Under standard conditions, 10-100 photons per atom can be scattered in a measurement time of $10 \mu$ s without blurring the image on the scale of $10 \mu \mathrm{m}$ [18], indicating that detection of atomic noise is possible. A typical cloud of $N=10^{7}{ }^{6} \mathrm{Li}$ atoms [21] will reach optical depth $\kappa \sim 1$ after expansion to about $1 \mathrm{~mm}$. Therefore, each $10 \mu \mathrm{m}$ bin will contain about $10^{3}$ atoms. The atomic noise contribution will cause bin-to-bin variation in the optical absorption at the level of few percent, which is detectable with current technology.
We now verify that in the case of a fermionic superfluid, the correlated fluctuations rise, in a statistical sense, above the background. For fixed $\mathbf{r}$, the value of $\Delta n\left(\mathbf{r}, \mathbf{r}^{\prime}\right)^{2}$ will exhibit fluctuations of order $n_{s}(r)$ as we vary $\mathbf{r}^{\prime}$. Therefore, the dip at $\mathbf{r}+\mathbf{r}^{\prime}=0$, also of magnitude $\sim n_{s}(r)$, may be observed only after averaging a number of such plots corresponding to independent choices of $\mathbf{r}$. However, different positions $\mathbf{r}$ yield independent results only if they correspond to distinct momenta in the trap. Thus, the number of independent positions on the two-dimensional image, is limited by momentum quantization in the trap to $O\left(N^{2 / 3}\right)$, where $N$ is the total number of atoms. Consequently the background statistical fluctuations can be averaged to $N^{-1 / 3}$ of the dip magnitude making it observable in a single image measurement. Similar considerations apply to Mott states in an optical lattice.

In conclusion, we demonstrated that spatial noise correlations in the image of an expanding gas cloud released from a trap, can reveal key properties of strongly correlated states of cold atoms. We anticipate that a similar technique can be used to observe signatures of more exotic states such as spin liquids and valence bond solids.

Note added in proof. Recently, experimental results closely related to the theoretical proposal described here were reported by Hadzibabic et al. [22].

We acknowledge valuable discussions with I. Bloch, M. Greiner, M. Prentiss and W. Ketterle. This work was supported by ARO, NSF (PHY-0134776, DMR-0132874), Sloan Foundation, and Packard Foundation.
[1] M. H. Anderson et al., Science 269, 198 (1995).

[2] M. R. Andrews et al., Science 275, 637 (1997).

[3] M. Greiner, O. Mandel, T. Esslinger, T. W. Hänsch, and I. Bloch, Nature (London) 415, 39 (2002).

[4] A. G. Truscott et al., Science 291, 2570 (2001); T. Loftus et al., Phys. Rev. Lett. 88, 173201 (2002); K. M. Ohara et al., Science 298, 2179 (2002); T. Bourdel et al., Phys. Rev. Lett. 91, 020402 (2003); S. Gupta et al., Science 300, 1723 (2003).

[5] M. Houbiers et al., Phys. Rev. A 56, 4864 (1997); M. Holland et al., Phys. Rev. Lett. 87, 120406 (2001); E. Timmermans, Phys. Lett. A 285, 228 (2001).

[6] E. Demler and F. Zhou, Phys. Rev. Lett. 88, 163001 (2002).

[7] A. B. Kuklov and B. V. Svistunov, Phys. Rev. Lett. 90, 100401 (2003).

[8] L.-M. Duan, E. Demler, and M. D. Lukin, Phys. Rev. Lett. 91, 090402 (2003); E. Altman et al., New J. Phys. 5, 113 (2003).

[9] A. Recati et al., J. Opt. B: Quantum Semiclassical Opt. 5, S55 (2003); B. Paredes and J. I. Cirac, Phys. Rev. Lett. 90, 150402 (2003).

[10] Ya. M. Blanter and M. Buttiker, Phys. Rep. 336, 1 (2000).
[11] See, e.g., M. O. Scully and M. S. Zubairy, Quantum Optics (Cambridge University Press, Cambridge, 1997).

[12] M. Randeria, in Bose-Einstein Condensation, edited by A. Griffin, D. W. Snoke, and S. Stringari (Cambridge University Press, Cambridge, 1995); A. J. Leggett, J. Phys. (Paris), Colloq. 41, C7 (1980).

[13] C. Menotti, P. Pedri, and S. Stringari, Phys. Rev. Lett. 89, 250402 (2002).

[14] W. Hofstetter et al., Phys. Rev. Lett. 89, 220407 (2002).

[15] W. Zhang, C. A. Sackett, and R. G. Hulet, Phys. Rev. A 60, 504 (1999).

[16] P. Torma and P. Zoller, Phys. Rev. Lett. 85, 487 (2000).

[17] D. Hellweg et al., Phys. Rev. Lett. 91, 010406 (2003).

[18] W. Ketterle, D. S. Durfee, and D. M. Stamper-Kurn, e-print cond-mat/9904034.

[19] R. Roth and K. Burnett, Phys. Rev. A 67, 031602(R) (2003).

[20] W. Oliver et al., Science 284, 299 (1999).

[21] Z. Hadzibabic et al., Phys. Rev. Lett. 91, 160401 (2003).

[22] Z. Hadzibabic et al., e-print quant-ph/0405113. 